\title{
Parsing Physiological Functions of Erythropoietin One Domain at a Time
}

\author{
Lawrence Steinman ${ }^{1}$
}

Published online: 27 August 2015

(C) The American Society for Experimental NeuroTherapeutics, Inc. 2015

\begin{abstract}
A domain of erythropoietin (EPO), separate from the domain involved in red blood cell development, has been identified. This region of EPO has anti-inflammatory and neuroprotective effects. Use of a peptide sequence from this region provides the potential for an effective therapeutic without effects on erythropoiesis.
\end{abstract}

Keywords Erythropoietin $\cdot$ Neuroprotection $\cdot$ Multiple sclerosis $\cdot$ Experimental autoimmune encephalomyelitis

Although erythropoietin (EPO) is best known for its role in hematopoiesis, there are other functions that are instigated via this protein. EPO is effective as a neuroprotective molecule in various animal models of neuroinflammation and ischemia, including experimental autoimmune encephalomyelitis (EAE), brain trauma, and stroke [1-6]. Fortunately, proteins are often divided into discrete domains with different functional activities residing in different regions of the molecule. In this issue of Neurotherapeutics, Yuan et al. [7] discuss how they were able to dissociate a domain of EPO with antiinflammatory and neuroprotective effects from the regions of the molecule responsible for erythropoiesis. The ability to isolate the neuroprotective and anti-inflammatory effects of the molecule from its effects on red blood cell (RBC) development might enable the design of potent guardian molecules

This comment refers to the article available at: http://dx.doi.org/10.1007/ s13311-015-0379-1.

Lawrence Steinman

steinman@stanford.edu

1 Neurology and Neuroscience, Stanford University School of Medicine, Stanford, CA 94305, USA for unmet neurologic needs, without the potential side effect of EPO causing polycythemia, hypertension, and stroke.

Yuan et al. [7] devised a successful strategy to parse the domains of EPO that might be free of its effect on RBC mass, while still retaining its neuroprotective effects. They made a library of peptides of lengths ranging from 7 to 25 amino acids, containing 1-2 cysteines. A 19-mer peptide containing 2 cysteines, termed JM-4, gave the strongest results, with antiinflammatory and protective properties dissociated from effects on RBC mass. The JM-4 peptide is derived from the AB loop of EPO. Shorter linear peptides, and peptides without the cysteines, were far less effective. For example, a 17-mer peptide from the same region was far less effective [7].

The diligence and persistence of Yuan et al. [7] were worthwhile: they learned that while the 19-mer JM-4 was effective in 2 models of EAE, they also discovered that a shorter 17-mer containing only 1 cysteine residue was far less effective in these models. Apparently, length mattered considerably in this case: incorporation of the second cysteine was critical for these protective effects. Further understanding of the structure/function relationships stemming from this observation is likely to be interesting and will illuminate how EPO interacts with its receptor to attenuate inflammation.

Yuan et al. [7] were able to demonstrate the reversal of paralytic disease at the onset of symptoms without effects on RBC mass. Proinflammatory cytokine production was reduced. JM-4 protected against demyelination and axonal loss [7]. In addition, they showed that JM-4 protected from amyloid $\beta$-induced toxicity in an in vitro model, indicating that this molecule might have protective properties independent of its anti-inflammatory roles.

Receptors for EPO are positioned at the blood-brain barrier, and we might assume that JM-4 reaches the brain via these receptors, just as native EPO does [2]. This issue will be resolved by further experimentation. There are other molecules with unexpected roles present at the blood-brain barrier. For 
example, in lesions of multiple sclerosis, elements of the clotting cascade, normally found at the brain endothelial interface, were discovered with laser capture microdissection and proteomic analysis. This research indicated a potential role in multiple sclerosis for activated protein $\mathrm{C}$, one of the main anticoagulants in human physiology [8]. Han et al. [8] were able to dissociate the anticoagulant activities of activated protein $\mathrm{C}$ from its anti-inflammatory activities in EAE. The division of these activities into different domains of activated protein $\mathrm{C}$ allowed Han et al. [8] to engineer versions of this protein with the desired anti-inflammatory characteristics without its undesirable side effects on the coagulation cascade.

As we learn more about protein structure we are recognizing that discrete structural domains in proteins have discrete functions. It seems that proteins may have evolved as if they were the result of deliberation of some "committee process", found in successful social enterprises. The "committee process" here is merely evolution.

We have learned that in some cases the domains of proteins are also spatially flexible, changing positions to accomplish different functions. This biologic strategy is actually employed in certain proteins with enzymatic activity. For example, fatty acid synthases are highly flexible proteins, where different domains actually move to accomplish interaction of the substrate with a key portion of the enzyme [9]. Molecular motors like dynein may have individual domains that are critical for certain tasks as they transport cargo down axons [10].

In some cases for protein engineering of a given function, it is possible to merely eject a module that is unnecessary, much like a spacecraft can jettison its earlier stages, when it is no longer needed. Like the rockets designed by those famous "rocket scientists", proteins can be designed by "protein engineers" without some of the undesired features, to make a new generation of guardian molecules [11]. It appears that EPO and activated protein C are 2 proteins that can be designed to provide anti-inflammatory benefit, without unwanted side effects. In the case of EPO, it appears that JM-4 is the critical module for attenuating inflammation and that other portions of this 165 -amino acid molecule can be jettisoned. Someday, we may see that the neuroengineers at "protein mission control" might prescribe JM-4 for a successful payload for conquering neurodegeneration and neuroinflammation.

Acknowledgments I thank Stanford University and the Zimmermann Chair allowing discretionary time to write and to think. There is no other financial support associated and no conflicts of interest.

Required Author Forms Disclosure forms provided by the authors are available with the online version of this article.

\section{References}

1. Sakanaka M, Wen TC, Matsuda S, et al. In vivo evidence that erythropoietin protect neurons from ischemic damage. Proc Natl Acad Sci U S A 1998;95:4635-4640.

2. Brines ML, Ghezzi P, Keenan S, et al. Erythropoietin crosses the blood-brain barrier to protect against experimental brain injury. Proc Natl Acad Sci U S A 2000;97:10526-10531.

3. Buemi M, Cavallaro E, Floccari F, et al. The pleiotropic effects of erythropoietin in the central nervous system. J Neuropathol Exp Neurol 2003;62:228-236.

4. Wang Y, Zhang ZG, Rhodes K, et al. Post-ischemic treatment with erythropoietin or carbamylated erythropoietin reduces infarction and improves neurological outcome in a rat model of focal cerebral ischemia. Br J Pharmacol 2007;151:1377-1384.

5. Li W, Maeda Y, Yuan RR, et al. Beneficial effect of erythropoietin on experimental allergic encephalomyelitis. Ann Neurol 2004;56: 767-777.

6. Meng Y, Xiong Y, Mahmood A, et al. Dose-dependent neurorestorative effects of delayed treatment of traumatic brain injury with recombinant human erythropoietin in rats. J Neurosurg 2011;115:550-560.

7. Yuan RR, Wang B, Lu W, et al. A distinct region in erythropoietin that induces immuno/inflammatory modulation and tissue protection. Neurotherapeutics 2015. doi:10.1007/s13311-015-0379-1.

8. Han MH, Hwang S, Roy DB, et al. Proteomic analysis of active multiple sclerosis lesions reveals therapeutic targets. Nature 2008;451:1076-1081.

9. Maier T, Leibundgut M, Ban N. The crystal structure of a mammalian fatty acid synthase. Science 2008;321:1315-1322.

10. Pfister KK. Distinct functional roles of cytoplasmic dynein defined by the intermediate chain isoforms. Exp Cell Res 2015;334:54-60.

11. Steinman L. No quiet surrender: molecular guardians in multiple sclerosis brain. J Clin Invest 2015;125:1371-1378. 